\title{
Amniale AFP-Konzentration in Schwangerschaften mit
} numerischen Chromosomenaberrationen

\begin{tabular}{|l|l|l|}
\hline E. & Elisabeth & Vytiska-Binstorfer \\
\hline H. & Heidrun & Genger \\
\hline J. & & Huber \\
\hline
\end{tabular}

I. Univ.-Frauenklinik Wien (Vorstand: Prof. Dr. E. Gitsch)

Dr. Elisabeth Vytiska-Binstorfer, I. Univ.-Frauenklinik Wien, Spitalgasse 23, A-1090 Wien

In einer retrospektiven Arbeit wurde das Alpha-Fetoprotein (AFP) aus dem Frucht-wasser von Schwangerschaften mit numerischen Aberrationen mit einer Normkurve vergli-chen, welche an Hand von AFP-Werten gesunder Schwangerer erstellt worden war. Zwi-schen 15. und 23. Schwangerschaftswoche wurden insgesamt 360 AFP-Konzentrationen in den verschiedenen Gestationswochen evaluiert und an Hand der Mittelwerte dieser 360 normalen Schwangerschaften wurden eine AFP-Normkurve erstellt (Abb. 1). Diese Ver-<smiles>IC(I)(I)C(I)(I)C(I)(I)C(I)(I)C(I)(I)C(I)(I)C(I)(I)C(I)(I)I</smiles>

o 1 risomif $18 \cdot$ Triiomic 21

Of-Fftoprotein im FW (ng/m!

30-

20-

$10-$

"-r-

15

$\sim 1-$

16

17 


$$
\begin{aligned}
& -\mathrm{t}-18 \\
& -\mathrm{i}- \\
& 19 \\
& 20 \\
& -\mathrm{r} \sim 21 \\
& -\mathrm{f}-22 \\
& -, \\
& 23 \mathrm{SSW}
\end{aligned}
$$

Abb. 1. Normkurve von AFP im Fruchtwasser normaler Schwangerschaften $(n=360)$.

Freie Vorträge (Geburtshilfe: Schwangerschaft)

139

laufskurve bestätigte die physiologische AFP-Konzentrationsabnahme zu Beginn des 2. Gestationstrimenons [3].

Bei 25 Patientinnen (1,9\%) von insgesamt 1330 Patientinnen wurde eine autosomale Trisomie festgestellt und deren AFP-Werte wurden mit jenen der 360 normalen Schwanger-schaften in Beziehung gesetzt.

Die AFP-Bestimmung wurde unmittelbar nach der Amniozentese mittels Radioimmu-noassay durchgeführt, welcher eine Sensibilität von weniger als $10 \mathrm{ng} / \mathrm{ml}$ aufweist.

Von den 25 Patientinnen mit numerischer Aberration zeigten 19 einen AFP-Wert unterhalb des Mittelwertes - allerdings in 14 Fallen noch innerhalb der Standardabweichung, bei 5

Patientinnen befand er sich darüber (Abb. 1).

Niedrige AFP-Werte im Fruchtwasser von Schwangerschaften mit Trisomie 21 bzw. 18 wurden wiederholt beschrieben $[1,2]$. In unseren Untersuchungen konnten wir ebenfa \s eine, allerdings nicht so ausgeprägte Erniedrigung der AFP-Werte feststellen. 5 Patientinnen (20\%) zeigten immerhin einen Wert, der deutlich über dem Mittelwert lag.

Literatur

Cuckle, H. S., Wald, N. J, Lindenbaum, R. H., Jonasson, J.: Amniotic Fluid AFP Levels and Down Syndrome. The Lancet; p. 290-91 (1985).

Dickermann, L. H., Berger, D. E., Johnson, W. E.: Low Alpha-Fetoprotein levels in pregnancies involving autosomal anueploidy. Am. J. Human Genetics, 37, 4, Suppl. A. 215/638 (1985). Hullin, D. A., Gregory, P. J.,Dyer, C. L., Dew, J. O.: Place of amniotic fluid AFP 1/8 prenatal diagnosis of trisomies. The Lancet, p. 662 (1985).

Gynäk. Rdsch. 26: suppl. 2, pp. 139-141 (1986)

Rasche Bestimmungsmethoden für LH aus Harnproben

Ch. Bieglmayer, F. Fischl

II. Univ.-Frauenklinik Wien (Vorstand: Prof. Dr. H. Janisch)

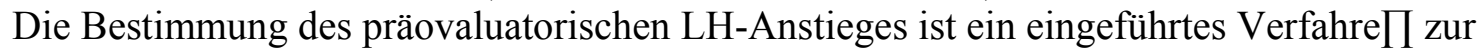
Eingrenzung der optimalen Konzeptionszeit. Insbesondere bei stimulierten Zyklen, die eine durch Clomiphen verminderte Zervixfunktion aufweisen können oder bei geplante $\prod$ intrauterinen Inseminationen, ist eine Kenntnis des Konzeptionsoptimums erwünscht. Bei 